\title{
Dynamic Viscoelastic Properties of ABS Polymers in the Molten State IV. Effect of Rubber Particle Size
}

\author{
Yuji AOKI and Kenjiro NAKAYAMA \\ Yokkaichi Research and Development Department, Mitsubishi Monsanto \\ Chemical Co. Ltd., Toho-cho, Yokkaichi, Mie 510, Japan
}

(Received June 17, 1982)

\begin{abstract}
Dynamic viscoelastic behavior of molten ABS polymers in which dispersed rubber particles are narrow in size distribution and hardly agglomerate were investigated as a function of the rubber content and the rubber particle size over wide ranges of temperature and frequency. The relaxation of the matrix AS copolymer associated with chain entanglement shifted the relaxation spectra of ABS to a longer time in proportion to the volume fraction of the dispersed phase. On the other hand, the long time relaxation spectra associated with particle-particle interactions depended strongly on both rubber content and particle size. With an increase in the rubber content and also a decrease in the rubber particle size, the spectra became higher. This may be explained as a result of a decrease in the surface distance between neighboring rubber particles. KEY WORDS ABS Polymer Melt / Viscoelasticity / Relaxation Spectrum / Long Time Mechanism / Particle Size Effect / Surface Distance /
\end{abstract}

Studies on the mechanical behavior of rubbermodified polymers have been concerned with dynamic properties, ${ }^{1-7}$ steady state viscosity, ${ }^{8-16}$ stress relaxation,,${ }^{9,17,18}$ and so on. ${ }^{7,16,19,20}$ It was found that the addition of rubber particles to a matrix polymer increases both the storage shear modulus $G^{\prime}$ and the loss shear modulus $G^{\prime \prime}$ and that the pseudo-equilibrium modulus $\left(G_{\mathrm{e}}\right)$ is attained at a low frequency. ${ }^{1-3}$ Corresponding to these changes in dynamic behavior, the increase in viscosity at small shear rates becomes more pronounced at higher rubber concentrations and smaller particle sizes and the yield phenomenon occurs as in other polymer-filler systems. ${ }^{21} \mathrm{Re}-$ cently, Münstedt ${ }^{16}$ demonstrated that the yield stress is caused by agglomeration of rubber particles and proposed two different models for the occurrence of such a stress.

We found that there are two kinds of ABS polymers, each with different dynamic behavior. ${ }^{3}$ One kind has a nonzero $G_{\mathrm{e}}$ and was found by Zosel, ${ }^{1}$ and Moroni and Casale $^{2}$; the other has no $G_{\mathrm{e}}$ and its $G^{\prime}$ decreases with a lowering of frequency. The former was found in the systems in which rubber particles agglomerate in the matrix phase, while the latter was found in finely dispersed sys- tems. It was also found that the viscoelastic properties of ABS polymers are influenced by acrylonitrile content $(\mathrm{AN} \%$ ) in grafted acrylonitrile-styrene (AS) copolymers. ${ }^{6}$ When the difference in $\mathrm{AN} \%$ between grafted and matrix AS copolymers was small, the rubber particles were dispersed finely without agglomeration, and no $G_{\mathrm{e}}$ was found. The mismatching of $\mathrm{AN} \%$ caused the agglomeration of particles and led to the observation of $G_{\mathrm{e}}$ in these systems. Thus, the rheological properties of molten ABS polymers are strongly affected by the agglomeration of particles.

Zosel $^{1}$ and Münstedt ${ }^{16}$ studied the effects of particle size on rheological behavior using rubber agglomerated systems. In these systems, the rheological behavior was affected not only by particle size, but also by particle agglomeration. The particle size distribution in ABS polymers is generally very broad or bimodal. Therefore, the data of these authors are not considered suitable for quantitative analysis. For this purpose, it is necessary to use ABS polymers, in which rubber particles have a narrow size distribution and incur no agglomeration. 


\section{EXPERIMENTAL}

Two series of ABS polymers (ABS-C and ABS$D)$ were used in this work. Figure 1 shows the electron microscopic photographs of rubber latices in ABS-C and ABS-D prior to graft polymerization. These photographs show the presence of very uniform spheres. Figure 2 shows the histograms of particle diameter for the two samples. It can be seen that these samples have very narrow distributions of rubber particle size. The number-average particle diameters for ABS-C and ABS-D were 350 and $170 \mathrm{~nm}$, respectively.

Table I shows some characteristics of the ABS polymers used in this work. The grafting degree (defined as the weight of grafted AS copolymer relative to that of rubber particles) was 0.20 and 0.41 for ABS-C and ABS-D, respectively. ABS polymers with different rubber content were prepared by blending AS copolymers with ABS

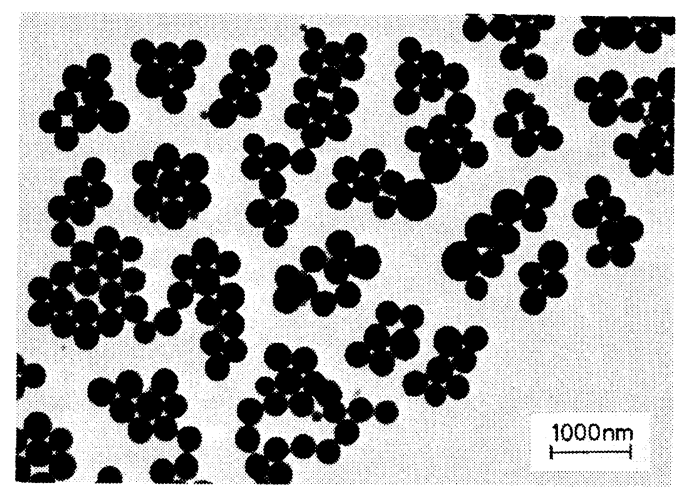

(a)

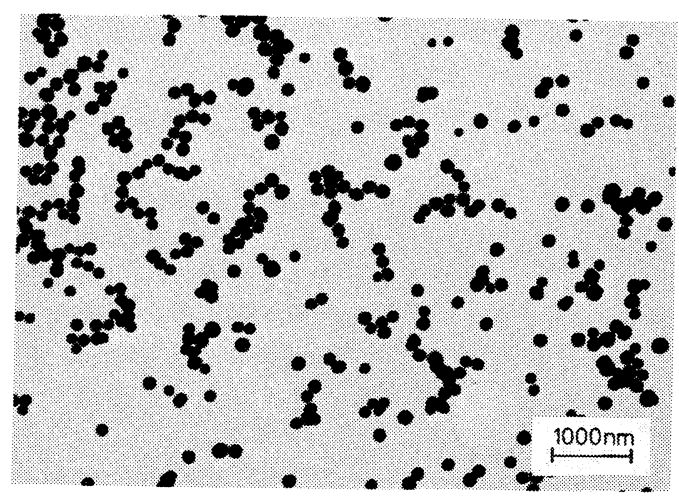

(b)

Figure 1. Electron microscopic photographs of rubber latices of ABS-C(a) and ABS-D(b). polymers of high rubber content. Blending was performed at $200^{\circ} \mathrm{C}$ for about 10 min using a Brabender Plastograph. The rubber content was 0 , $10,15,20,25$, and $30 \mathrm{wt} \%$ for ABS-C and 0, 5, 10, 15 , and $20 \mathrm{wt} \%$ for ABS-D.

The weight-average molecular weight of the matrix AS copolymer was obtained from the intrinsic viscosity in $N, N$-dimethylformamide at $20^{\circ} \mathrm{C}$, using the equation of Lange and Baumann. ${ }^{22}$ All the samples used had almost the same molecular weight of 57,500 . The polydispersity index $M_{w} / M_{n}$ was estimated by GPC to be 2.7. The AN\% of the AS copolymer was $25.7 \mathrm{wt} \%$. The $\mathrm{AN} \%$ and molecular

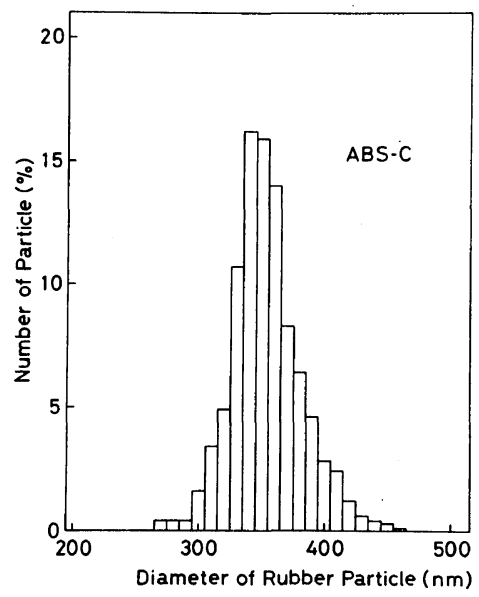

(a)

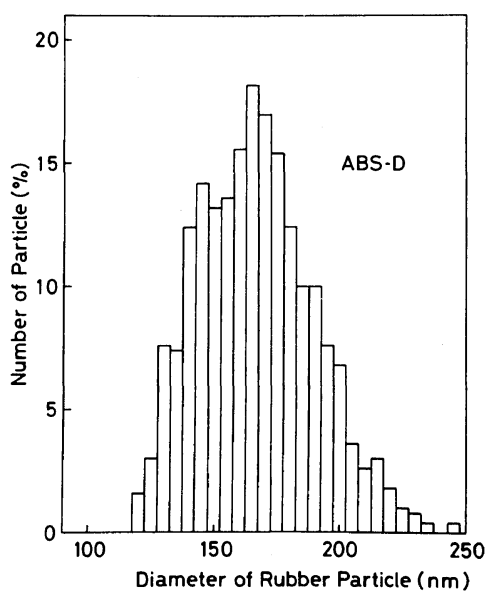

(b)

Figure 2. Histograms of particle diameter in ABS-C(a) and ABS-D(b) 
Table I. Sample codes and characteristics of ABS polymers ${ }^{\mathrm{a}}$

\begin{tabular}{|c|c|c|c|c|c|}
\hline \multirow{2}{*}{ Code } & \multirow{2}{*}{$\mathrm{Ru} \%$} & \multirow{2}{*}{$\phi$} & R.P.S. & $T_{\mathrm{s}}$ & $S$ \\
\hline & & & $\mathrm{nm}$ & ${ }^{\circ} \mathrm{C}$ & $\mathrm{nm}$ \\
\hline AS-1 & 0 & 0 & - & 130 & - \\
\hline C-10 & 10 & 0.138 & 350 & 131 & 208 \\
\hline C-15 & 15 & 0.204 & 350 & 132 & 138 \\
\hline C-20 & 20 & 0.270 & 350 & 133 & 91 \\
\hline C-25 & 25 & 0.334 & 350 & 134 & 63 \\
\hline C-30 & 30 & 0.397 & 350 & 134 & 39 \\
\hline D-5 & 5 & 0.079 & 170 & 131 & 166 \\
\hline D-10 & 10 & 0.158 & 170 & 132 & 93 \\
\hline D-15 & 15 & 0.233 & 170 & 133 & 57 \\
\hline D-20 & 20 & 0.308 & 170 & 134 & 35 \\
\hline
\end{tabular}

a $\mathrm{Ru} \%$, weight fraction of dispersed rubber; $\phi$, volume fraction of the dispersed phase (rubber + grafted AS copolymer); R.P.S., rubber particle size; $T_{\mathrm{s}}$, reference temperature obtained from the WLF equation; $S$, surface distance between neighbouring particles.

weight of the grafted AS copolymer were measured after ozonolysis of the acetone insoluble part of the ABS polymers. The AN\% of the grafted AS copolymer was nearly equal to that of the matrix AS copolymer. The $M_{w}$ of the grafted AS copolymer was somewhat larger than that of the matrix in ABS-C and ABS-D.

Measurement of viscoelastic properties was carried out with the concentric cylinder type rheometer using the method described previously. ${ }^{3}$ The temperature for measurement ranged from 130 to $245^{\circ} \mathrm{C}$, and the frequency from $6.67 \times 10^{-4}$ to $1 \mathrm{~Hz}$. All measurements were performed under nitrogen so as to minimize oxidative degradation at high temperature.

\section{RESULTS AND DISCUSSION}

The data for the storage shear modulus $G^{\prime}$ and the loss modulus $G^{\prime \prime}$ of each sample could be superimposed by shifting them along the time scale, except for $G^{\prime}$ of C-30 and D-20 at high temperatures. The shift factor $a_{\mathrm{T}}$ for ABS-C is plotted against the temperature difference $\left(T-T_{\mathrm{s}}\right)$ in Figure 3. Here, the reference temperature $T_{\mathrm{s}}$ is 130,131 , 133, and 134 for AS-1, C-10, C-20, and C-30, respectively. See Table $\mathrm{I}$ for the $T_{\mathrm{s}}$ values of the other samples used. The dotted line in Figure 3 represents the WLF equation ${ }^{23}$ :

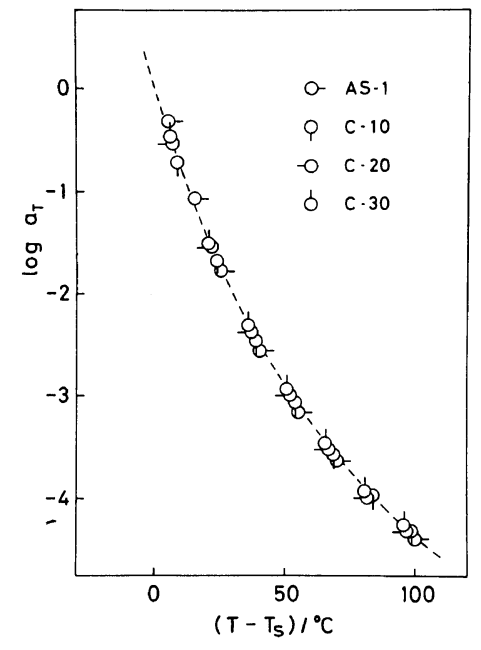

Figure 3. Shift factors $a_{\mathrm{T}}$ for AS-1, C-10, C-20, and C30 plotted against temperature difference $\left(T-T_{\mathrm{s}}\right)$. The reference temperature $T_{\mathrm{s}}$ is $130,131,133$, and 134 for AS-1, C-10, C-20, and C-30, respectively. The dotted line represents the WLF equation.

$$
\log a_{\mathrm{T}}=-8.86\left(T-T_{\mathrm{s}}\right) /\left(101.8+T-T_{\mathrm{s}}\right)
$$

The values of $T_{\mathrm{s}}$ increased slightly with the volume fraction of the dispersed phase, but was independent of the particle size. Previous work ${ }^{4,6,16}$ shows that $T_{\mathrm{s}}$ is independent of particle content. Therefore, a slight increase in $T_{\mathrm{s}}$ with volume fraction may be associated with the low molecular weight of the matrix AS copolymer.

Figures 4 and 5 show the master curves of $G^{\prime}$ and $G^{\prime \prime}$ for ABS-C with different rubber content at $170^{\circ} \mathrm{C}$. The corresponding curves for ABS-D are illustrated in Figures 6 and 7. Two characteristic rheological features of ABS polymers can be seen from these graphs.

First, the rubbery plateau regions of both $G^{\prime}$ and $G^{\prime \prime}$ shift to lower frequency with an increase in particle content. Second, in the region of low frequency, where $G^{\prime \prime}$ of the matrix AS copolymer exhibits a Newtonian flow, $G^{\prime}$ of the ABS polymers are little dependent on frequency, and the slopes of $G^{\prime \prime} v s$. frequency of the ABS polymers become smaller than unity with an increase in particle content.

Next, the master curves of ABS-C with those of ABS-D in the region of low frequency are compared. For ABS-C, the $G^{\prime \prime}$ curves for C-10, C-15, and $\mathrm{C}-20$ on the $\log -\log$ scale have slopes of about 


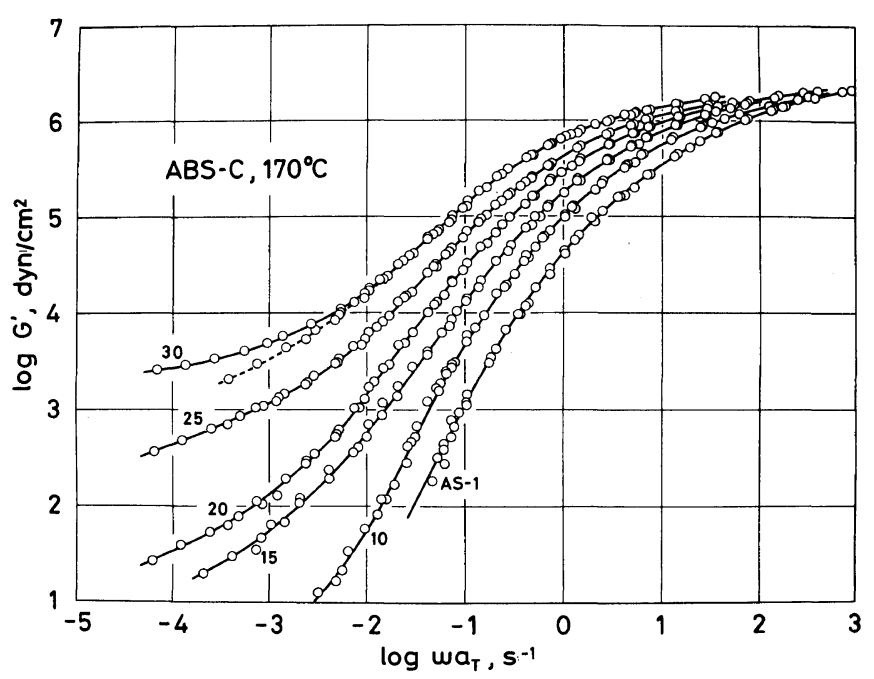

Figure 4. Master curves of storage shear modulus $G^{\prime}$ for ABS-C with different rubber content at $170^{\circ} \mathrm{C}$.

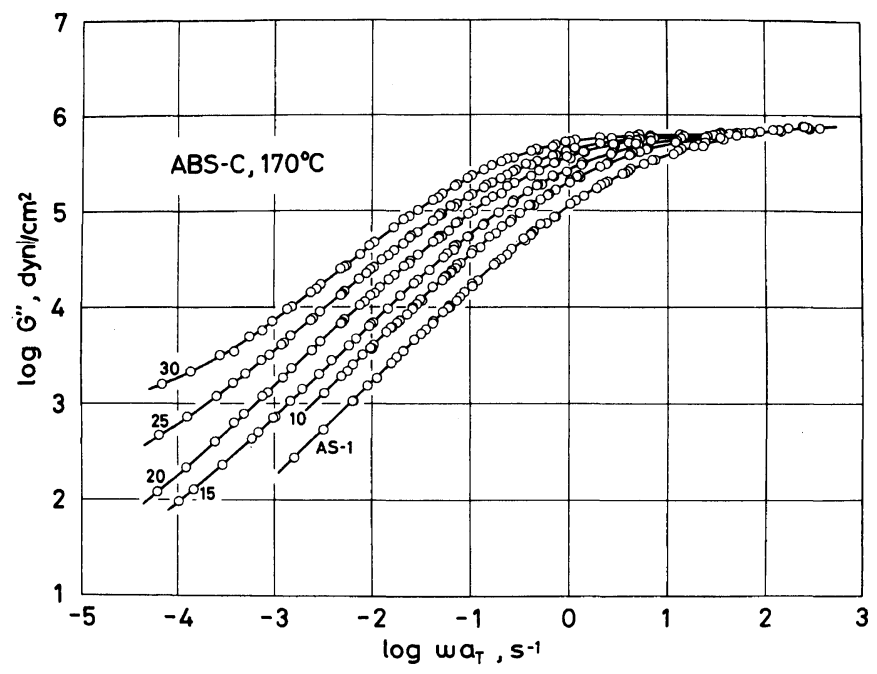

Figure 5. Master curves of loss modulus $G^{\prime \prime}$ for ABS-C with different rubber content at $170^{\circ} \mathrm{C}$.

unity. In other words, these samples have zero shear viscosity. On the other hand, the slopes of the $G^{\prime \prime}$ curves for ABS-D are smaller than unity even at a $15 \%$ rubber content, although D-5 and D-10 have zero shear viscosity. Rubber particles in both ABS$\mathrm{C}$ and ABS-D are dispersed finely without agglomeration. In such cases, a zero shear viscosity is obtained even for ABS polymers in which particle sizes are less than one micron, as in the case of high impact polystyrene (HIPS) containing large particles. ${ }^{19,20}$ However, no zero shear viscosity was obtained even at a $10 \%$ rubber content in the case of rubber agglomerated ABS polymers. ${ }^{3}$

We calculated the surface distance $S$ between neighbouring particles, making the assumption that the particles are arranged in a cubic lattice and covered uniformly with grafted AS copolymers $(15 \mathrm{~nm}$ thick for ABS-C and $10 \mathrm{~nm}$ thick for ABSD). The value of $S$ for each sample is listed in Table I. When $S$ is larger than about $90 \mathrm{~nm}$ on the average, a zero shear viscosity was obtained for both ABS-C and ABS-D. It may be considered that 


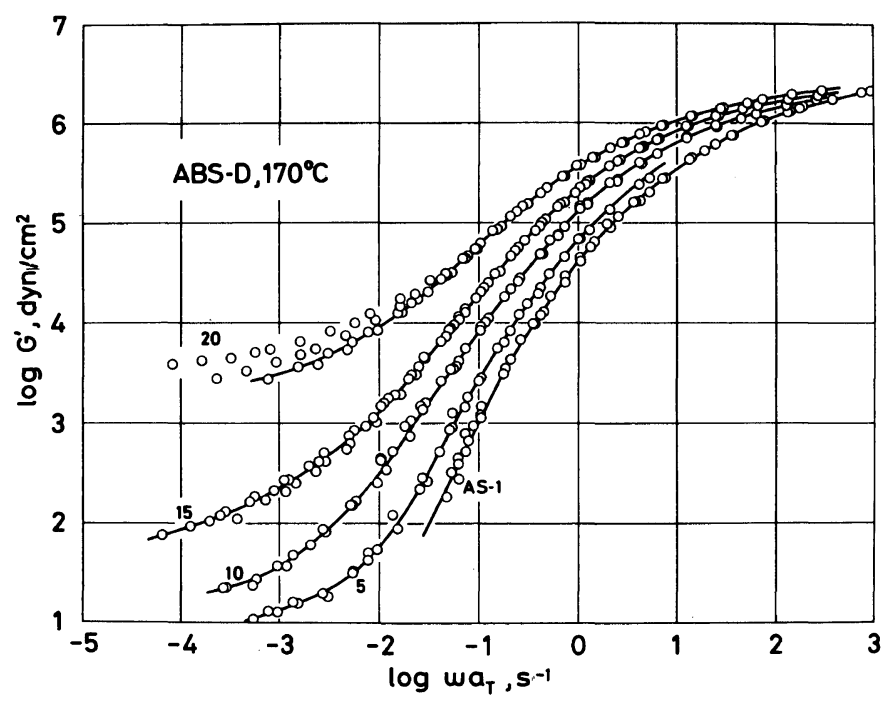

Figure 6. Master curves of storage shear modulus $G^{\prime}$ for ABS-D with different rubber content at $170^{\circ} \mathrm{C}$.

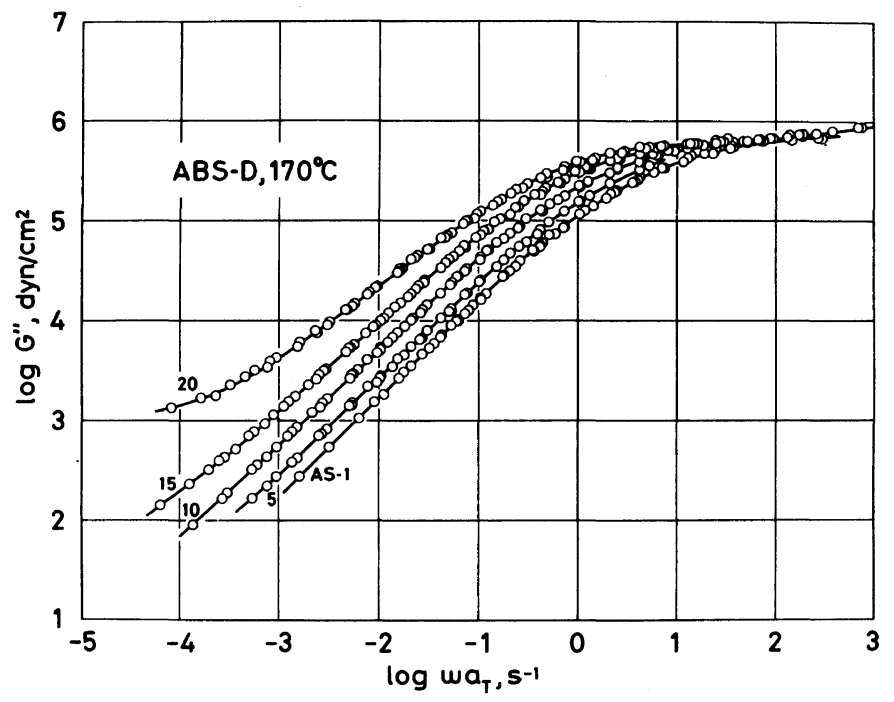

Figure 7. Master curves of loss modulus $G^{\prime \prime}$ for ABS-D with different rubber content at $170^{\circ} \mathrm{C}$.

the interaction between particles becomes stronger as the surface distance becomes smaller, leading to the disappearance of zero shear viscosity.

The relaxation spectra $H(\tau)$ of ABS polymers were calculated using the Tschoegl equation. ${ }^{24}$ The results for ABS-C and ABS-D are shown in Figures 8 and 9 , respectively. The large circles were obtained from the master curves of $G^{\prime}$, and the small ones from those of $G^{\prime \prime}$. The two sets of data points are in good agreement with each other. The relaxation spectra of ABS polymers were separated in two regions, ${ }^{5}$ one associated with the entanglement of AS chains and the other with the polymers at long relaxation time. Figure 10 shows the volume fraction dependence of $\tau / \tau_{\mathrm{AS}}$, the relaxation time of the AS copolymer in the ABS polymer relative to that of the matrix AS copolymer. The latter was evaluated by shifting the $H(\tau)$ of the matrix AS copolymer to those of the ABS polymers. The data points for both ABS-C and ABS-D fall on the same 


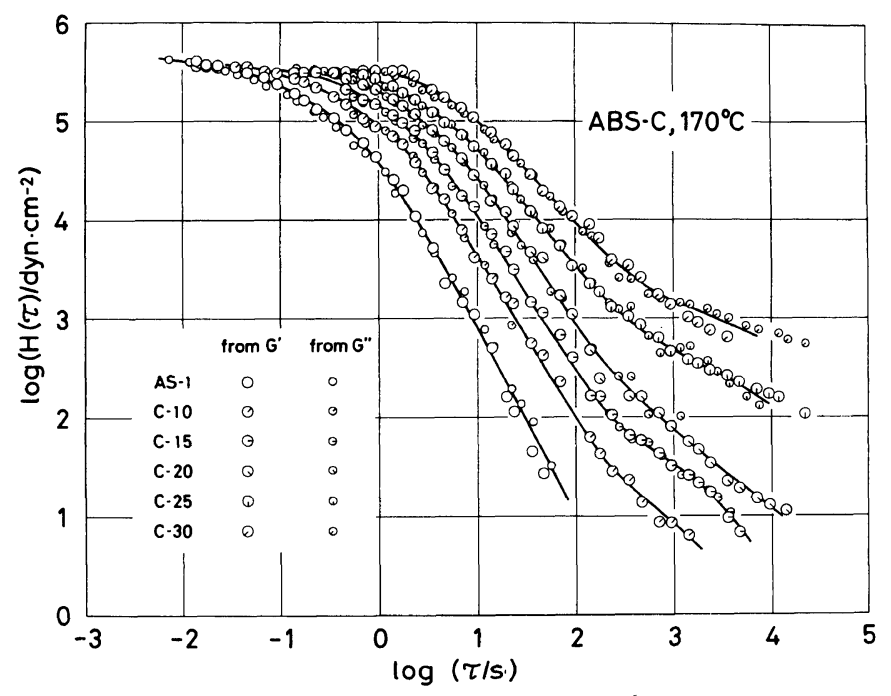

Figure 8. Relaxation spectra for ABS-C with different rubber content at $170^{\circ} \mathrm{C}$. Large circles were obtained from the master curves of $G^{\prime}$ and small circles from those of $G^{\prime \prime}$

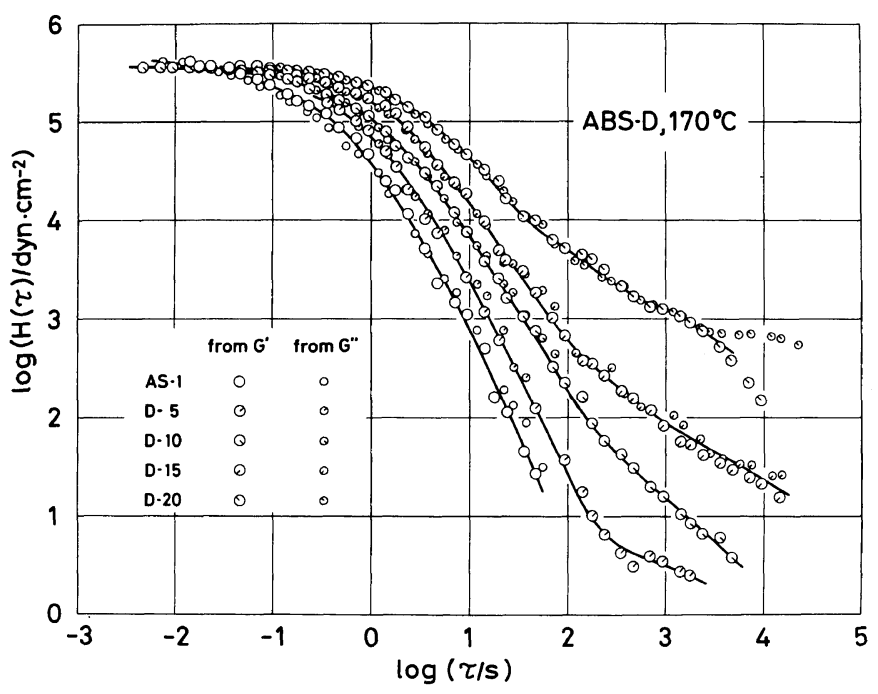

Figure 9. Relaxation spectra for ABS-D with different rubber content at $170^{\circ} \mathrm{C}$.

straight line having a slope of about 3 , thus yielding

$$
\tau=\tau_{\mathrm{AS}} \exp (7 \phi)
$$

where $\phi$ is the volume fraction of the dispersed phase. This relation is in agreement with the one reported previously by Aoki, ${ }^{5}$ and indicates that the relaxation spectrum of the continuous phase is shifted to longer time by dispersing grafted rubber particles. This shift depends only on the volume fraction of the dispersed phase and is independent of the rubber particle size.

It may be considered that $\tau / \tau_{\mathrm{AS}}$ is a measure of the viscosity increase due to the dispersion of particles in a Newtonian fluid, since the viscosity of such a system is determined by hydrodynamic interaction and hence should depend only on the volume fraction of the dispersed phase. However, our experimental volume fraction dependence of $\tau / \tau_{\mathrm{AS}}$ is 


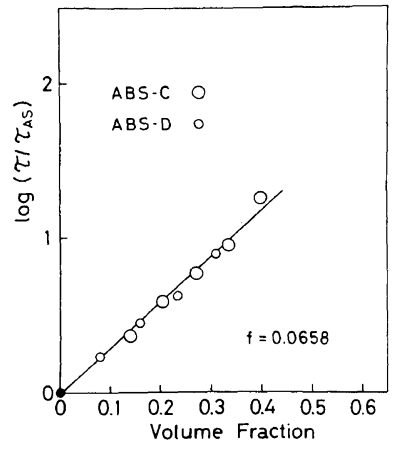

Figure 10. Volume fraction dependence of $\tau / \tau_{\mathrm{AS}}$ at $f=0.0658$.

somewhat different from the equations proposed in earlier papers (for example, Mooney's equation ${ }^{25}$ ). This discrepancy is not clear, but one reason may be the interaction between the matrix and the grafted AS copolymers near the surfaces of particles.

Next, the long time relaxation spectra characteristic of ABS polymers are discussed. These spectra depend strongly on the particle size. Figure 11 shows $H(\tau)$ at $\tau=10^{3} \mathrm{~s}$ logarithmically plotted against $S$ at the iso-free volume condition of $f=$ 0.0658 . The plotted points are fitted by a straight line with a slope of 3 for both ABS-C and ABS-D except for D-20. The long time relaxation spectra depend on $S$, if the particle sizes are equal. However, $H(\tau)$ for ABS-D is lower than that for ABS-C at a given $S$, indicating that $H(\tau)$ for large $\tau$ depends not only on $S$, but also on particle size. Hence, $H(\tau)$ at two values of $\tau$ were plotted against $S$ divided by the particle size $D$. The results shown in Figure 12 indicate that the long time relaxation spectra depend on both $S$ and $D$. At a constant relaxation time, $H(\tau)$ is proportional to $(S / D)^{-n}$. For $\tau=10^{3} \mathrm{~s}, n$ is equal to 3 . With an increase in $\tau, n$ becomes larger.

Masuda et al. ${ }^{4}$ proposed a mechanism for the long time relaxation spectrum, considering that the shear rate or the shear stress between neighbouring particles is $[1+(S / D)]$ times larger than the external shear rate or shear stress, because there exists no velocity gradient in the particles. According to their model, the relaxation time is only a few times longer than that of the matrix AS copolymer. However, the experimental relaxation times are much longer. If the distribution of $S$ is broad, the relaxation spectra are also broad.

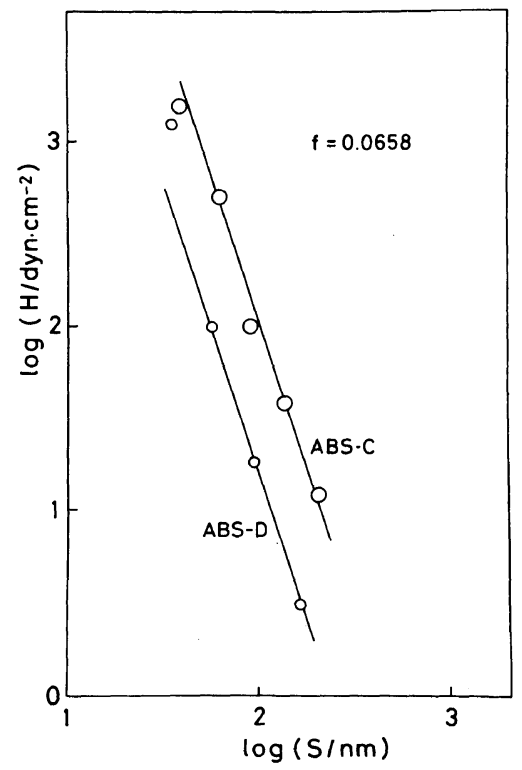

Figure 11. $H(\tau)$ at $\tau=10^{3} \mathrm{~s}$ logarithmically plotted against the surface distance between neighbouring particles $S$ for ABS-C and ABS-D at $f=0.0658$.

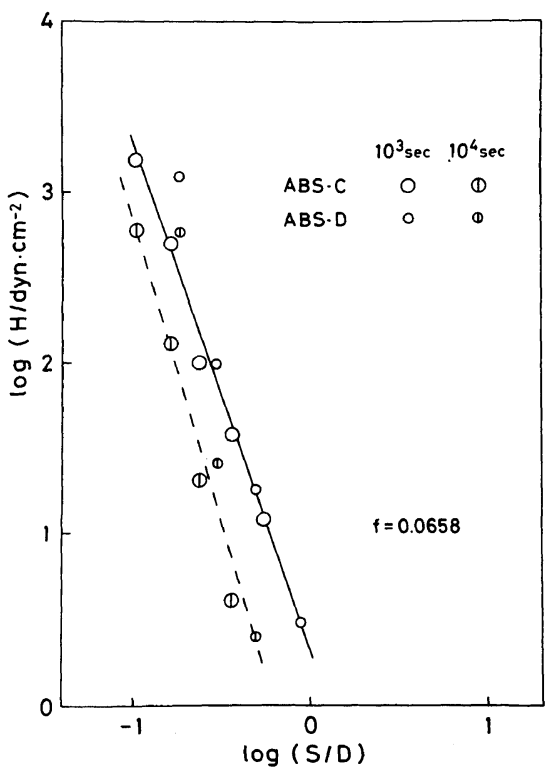

Figure 12. $H(\tau)$ at $\tau=10^{3}$ and $10^{4} \mathrm{~s}$ plotted against the surface distance between neighbouring particles $S$ divided by the particle size $D$ at $f=0.0658$.

The long time relaxation spectra may also be associated with the force acting between neighbouring particles (for example, entropic repulsive force 
or entanglement between grafted AS copolymers). In the long time region, the matrix AS copolymer is Newtonian and the $G^{\prime}$ is negligibly small. Hence, its elastic properties must be due to grafted particles. The smaller the surface distance between neighbouring particles, the stronger the interaction between these particles becomes. Moreover, the surface distance between neighbouring particles is distributed widely, as mentioned previously. Therefore, the relaxation spectra may be a function of both the surface distance and the particle size. It is hoped that an adequate theory will be developed to explain the mechanism of long time relaxation spectra.

The experimental results obtained may be summarized as follows. The rubbery plateau zone shifts to a longer time with an increase in rubber particles, depending only on the volume fraction of the dispersed phase. The long time relaxation spectra associated with particle-particle interactions depend strongly on the particle size and also on the surface distance between neighbouring particles.

\section{REFERENCES}

1. A. Zosel, Rheol. Acta, 11, 229 (1972).

2. A. Moroni and A. Casale, Proceedings of the 7 th International Rheology Congress, 339 (1976).

3. Y. Aoki, J. Soc. Rheol. Jpn., 7, 20 (1979).

4. T. Masuda, M. Kitamura, and S. Onogi, J. Soc. Rheol. Jpn., 8, 123 (1980).
5. Y. Aoki, J. Rheol., 25, 351 (1981).

6. Y. Aoki and K. Nakayama, J. Soc. Rheol. Jpn., 9, 39 (1981).

7. H. C. Booij and J. H. M. Palmen, Proceedings of the 8th International Rheology Congress, 501 (1980).

8. T. S. Lee, Proceedings of the 5th International Rheology Congress, 4, 421 (1970).

9. R. L. Bergen, Jr. and H. L. Morris, Proceedings of the 5th International Rheology Congress, 4, 524 (1970).

10. C. D. Han, J. Appl. Polym. Sci., 15, 2591 (1971).

11. A. Casale, A. Moroni, and C. Spreafico, Adv. Chem. Ser., 142, 172 (1975).

12. H. Kubota, J. Appl. Polym. Sci., 19, 2299 (1975).

13. R. A. Mendelson, Polym. Eng. Sci., 16, 690 (1976).

14. K. Itoyama and A. Soda, Kobunshi Ronbunshu, 31, 47 (1976).

15. K. Itoyama and A. Soda, J. Appl. Polym. Sci., 23, 1723 (1979).

16. H. Münstedt, Polym. Eng. Sci., 21, 259 (1981).

17. Y. Yoshitake, M. Fujiyama, S. Uemura, and M. Takayanagi, Kogyo Kagaku Zasshi, 71(8), 106 (1968).

18. K. C. Rusch, J. Macromol. Sci.-Phys., B2(3), 421 (1968).

19. R. L. Kruse and J. H. Southern, J. Rheol., 24, 755 (1980).

20. H. Tanaka and J. L. White, Polym. Eng. Rev., 1(1), 89 (1981).

21. For example, J. L. White, L. Czarnecki, and H. Tanaka, Rubber Chem. Technol., 53, 823 (1980).

22. H. Lange and H. Baumann, Angew. Makromol. Chem., 14, 25 (1970).

23. M. L. Williams, R. F. Landel, and J. D. Ferry, J. Am. Chem. Soc., 77, 3701 (1955).

24. N. W. Tschoegl, Rheol. Acta, 10, 583 (1971).

25. M. Mooney, J. Colloid Sci., 6, 162 (1951). 